some rationally connected system of hypotheses, and never wholly on direct observations. It is, naturally, in the latter parts of this book that the lack of a theoretical background, such as the economists have possessed for more than a century, is most severely felt. This criticism should not be taken as denying to the authors a great measure of success in an immensely arduous undertaking.

R. A. Fisher.

\section{The Endocrine Principles}

Recent Advances in Endocrinology. By Prof. A. T. Cameron. Pp. vii +365. (London : J. and A. Churchill, 1933.) $15 s$.

$7 \mathrm{HE}$ endocrine principles are of interest from three aspects, clinical, chemical and social, for there is often an attempt to ascribe differences of personality to their variations. Recently it is on the chemical side that most progress has been made, many of the principles having been isolated in pure crystalline form and even synthesised, so that it is possible to make the physiological study of the mechanism of their action with material of definite composition and structure. There is progress too on the clinical side, though these aspects are more a matter of controversy. Internal secretions are produced by the thyroid, parathyroid, pituitary and adrenal glands, by the islet tissues of the pancreas, the mucous membrane of the intestine and the organs of reproduction. From these there has been isolated thyroxine, adrenaline, insulin and ostrin in crystalline form, and there is presumptive evidence for other compounds.

Prof. Cameron gives six chapters to the wellestablished endocrine principles and one to the more presumptive ones, and devotes a final chapter to their interrelationships. $\mathrm{He}$ summarises what is known regarding their chemistry, their method of action, their physiology and their clinical behaviour. The book has an appeal, therefore, to the medical, as well as to the biochemical, expert. Each chapter has rather full references to the literature up to the end of 1932 and is appropriately illustrated.

Now that the structure of these compounds is known, it should be noted that they bear no relation to one another, and it becomes important to ascertain some clue as to their utility in metabolism. This is still largely guesswork, but there seems to be a hint of some general relation to oxidation phenomena.

The thyroid principle, for example, according to Plummer, exerts an influence on the oxidation proceeding in all the cells of the body and thus produces its actions : the behaviour suggests control of specific reactions. Insulin brings about the disappearance of glucose from the blood: it is undecided whether it facilitates glycogen formation or direct oxidation of glucose. Adrenaline likewise has an effect on the interconversion of glycogen and glucose. Of the less-known endocrines, secretin is the most interesting : it appears, like insulin, to be a protein, and acts to stimulate the outflow of pancreatic juice and bile. Mellanby considers that it is carried with the bile salts to the general circulation.

The author devotes a final short chapter to endocrine interrelationships, particularly those of the pituitary, which through one or other of the several principles it secretes controls (1) the thyroid and therefore the oxidative processes, (2) the adrenal cortex and thereby muscular contractility (3) the development of the gonads and hence the secondary sex organs and secondary sex characters, (4) fat metabolism to some degree, (5) the water exchanges of the body.

No additional words are required to emphasise the interest of the conception that crystallisable chemical substances of relatively simple composition are able to control so many effects in the organism, and it is easy to see the many ways in which slight variations in their production can be reflected. Further deductions from these facts soon take us outside the region of ascertained scientific facts and they are wisely eschewed by the author.

When a selection has to be made from so large a quantity of material, it would not be difficult to find faults of omission or over-emphasis, but it would be unfair to criticise the author for these when so much has been achieved in bringing a difficult subject into focus for detailed study. Prof. Cameron's book will be of definite help to all interested in this field.

\section{A New Atomic Model}

The Sub-Atoms: an Interpretation of Spectra in conformity with the Principles of Mechanics. By William Mayo Venable. Pp. viii +148 . (Baltimore, Md.: The Williams and Wilkins Co.; London: Baillière, Tindall and Cox, 1933.) $9 s$.

TTHIS book describes an attempt to explain 1 spectroscopic phenomena in terms of a model of the atom conforming to Newtonian mechanics. The author assumes the existence of 'sub-atoms', each of which consists of an ellipsoidal or similarly shaped mass of positive electricity, of charge $e$, on which rests a smaller negative electron (charge, $-e$ ). The linear dimensions of the sub-atoms are inversely as their masses, and the atom of any element consists of that particular stable association of sub-atoms which will explain simultaneously its mass, atomic volume and spectrum. For the 